\title{
Tanshinone-IIA inhibits myocardial infarct via decreasing of the mitochondrial apoptotic signaling pathway in myocardiocytes
}

\author{
YEQING FANG ${ }^{1,2}$, CHENGCHENG DUAN $^{3}$, SHAOYUAN CHEN $^{1}$, ZHENGUO LIU $^{4}$, \\ BIMEI JIANG ${ }^{4,5}$, WEN AI ${ }^{1}$, LEI WANG ${ }^{1}$, PEIYI XIE ${ }^{1}$ and HONGCHENG FANG ${ }^{3}$ \\ ${ }^{1}$ Department of Cardiology, Shenzhen Nanshan People's Hospital, Shenzhen, Guangdong 518000; \\ ${ }^{2}$ Shenzhen Nanshan Medical Group Headquarters, Shenzhen, Guangdong 518052; ${ }^{3}$ Department of Cardiology, \\ Shenzhen Hospital of Integrated Traditional Chinese and Western Medicine, Shenzhen, Guangdong 518000, \\ P.R. China; ${ }^{4}$ Dorothy M. Davis Heart and Lung Research Institute, The Ohio State University \\ Wexner Medical Center, Columbus, OH 51027, USA; ${ }^{5}$ Department of Burns and Plastic Surgery, \\ Xiangya Hospital, Central South University, Changsha, Hunan 410008, P.R. China
}

Received December 29, 2020; Accepted May 14, 2021

DOI: $10.3892 /$ ijmm.2021.4991

\begin{abstract}
Myocardial ischemia triggers an inflammatory reaction and oxidative stress that increases apoptosis of myocardiocytes. It has been evidenced that tanshinone-IIA (Tan-IIA) protects against heart failure post-myocardial infarction via inhibition of the apoptotic pathway. The purpose of the present study was to investigate the therapeutic effect of Tan-IIA in a rat model of myocardial ischemia, and explore the possible mechanism of Tan-IIA in myocardiocytes. The rat model of myocardial ischemia was established by left anterior descending coronary artery and rats received treatment with either Tan-IIA ( $10 \mathrm{mg} / \mathrm{kg}$ ) or PBS for 20 days continuously. The cardiac function in the experimental rat model was detected using the Sequoia 512 echocardiography system on day 21 . The cell viability of myocardiocytes was assessed by CCK- 8 assay. Apoptosis of myocardiocytes and myocardial tissue was evaluated by TUNEL assay. The infarct size of the myocardial ischemia rat was determined through 2,3,5-triphenyltetrazolium chloride (TTC) and Evan blue double staining assay. The expression levels of apoptotic factors were assessed by immunohistochemistry, western blotting and immunofluorescence. The results demonstrated that Tan-IIA reduced myocardial infarct size and improved the myocardial function in myocardial ischemia rats. Compared with PBS, Tan-IIA treatment decreased myocardial tissue apoptosis and the expression levels of
\end{abstract}

Correspondence to: Professor Hongcheng Fang, Department of Cardiology, Shenzhen Hospital of Integrated Traditional Chinese and Western Medicine, 3 Xinsha Road, Shenzhen, Guangdong 518000, P.R. China

E-mail: fangyeqing2005@hotmail.com

Key words: tanshinone-IIA, myocardial ischemia, myocardiocyte, apoptosis caspase-3, Cyto $c$ and Apaf-1 in myocardial tissue. Tan-IIA increased the viability of impaired myocardiocytes, inhibited apoptosis of impaired myocardiocytes and increased Bcl-2 and Bak expression in myocardiocytes. In addition, Tan-IIA increased Bim and CHOP, decreased TBARS, ROS and $\mathrm{H}_{2} \mathrm{O}_{2}$ production, decreased ATF4 and IRE1 $\alpha$ expression, and reduced intracellular calcium and oxidative stress in myocardiocytes. Furthermore, caspase-3 overexpression blocked Tan-IIA-decreased apoptosis of myocardiocytes. In conclusion, the data in the present study indicated that Tan-IIA improved myocardial infarct and apoptosis via the endoplasmic reticulum stress-dependent pathway and mitochondrial apoptotic signaling pathway.

\section{Introduction}

Myocardial ischemia disease remains a major cause of death $(\sim 1.72 \%)$ and disability $(\sim 2.45)$ in most countries in the world (1). Apoptosis of myocardiocytes can promote myocardial ischemia, ischemia/reperfusion (I/R) injury, post-ischemia cardiac remodeling and coronary atherosclerosis (2). Evidence has indicated that myocardial ischemia presents relatively high lethality, which is closely associated with metabolism disorders in endothelial cells of heart vessels (3). Numerous studies have indicated that the increase of myocardiocyte apoptosis contributes to the development of cardiovascular diseases (4-6). Foundationally, exploring drugs which protect against myocardial ischemia and reperfusion injury plays crucial a role in modulating myocardial apoptosis and levels of inflammation $(7,8)$. In addition, inhibition of myocardial ischemia injury-induced apoptosis of cardiomyocytes could significantly improve cardiac function (9). Furthermore, myocardial ischemia-reperfusion injury has been revealed to induce a sterile inflammatory response and apoptosis of myocardial tissue, which further contributes to the final infarct size (10). Therefore, developing new therapies for myocardial injury represents an urgent and significant research interest (11). 
Tanshinone-IIA (Tan-IIA) has been revealed to possess anti-atherosclerosis effects and is widely used in treatment of cardiovascular and cerebrovascular diseases (12). A previous study has reported that Tan-IIA demonstrates rich cardioprotective activities for clinical applications (13). Another study reported that Tan-IIA could inhibit the myocardial apoptosis in a heart failure rat model by upregulating the microRNA (miR)-133 level (14). In addition, Tan-IIA has been demonstrated to be an effective and safe agent for the treatment of patients with coronary heart disease (15). Furthermore, Tan-IIA has presented a significant protection of cardiomyocytes against apoptosis via decreasing oxidative stress and inflammatory responses (16). Although the anti-apoptotic effect of Tan-IIA has been well explored in animal models of myocardial ischemia (17), the molecular mechanism has not been clearly documented. Therefore, the effects and molecular mechanisms of Tan-IIA on cardiomyocytes were evaluated both in vitro and in vivo.

Oxidative stress is enhanced in chronic heart failure and is key to providing some suggestions for the treatment of heart diseases (18). Oxidative stress plays an important role in the pathophysiology of myocardial ischemia and improved understanding of the role of oxidative stress in myocardial ischemia resulted in novel therapeutic options for patients with myocardial ischemia (19). In addition, the mitochondrial pathway of apoptosis is activated in atrial fibrillation of heart failure patients, which contributes to the understanding of atrial contractile dysfunction (20). Furthermore, oxidative stress and the mitochondrial apoptotic pathway were revealed to be associated with apoptosis of cardiac myocytes induced by osteopontin (21). However, the precise mechanisms by which oxidative stress induces the mitochondrial apoptotic pathway in myocardiocytes remain unknown.

The purpose of this study was to investigate whether Tan-IIA had a protective effect on apoptosis of myocardial tissue in an animal model of myocardial ischemia. The potential anti-apoptotic mechanism of Tan-IIA in myocardiocytes was also investigated.

\section{Materials and methods}

Animals and drug treatment. The present study was approved (approval no. 20160512C10) by the Ethics Committee of Shenzhen Nanshan People's Hospital (Shenzhen, China). A total of 20 male Sprague Dawley (SD) rats (10 weeks old; 320-340 g body weight) were purchased from the Animal Experiment Center of Tongji University (Shanghai, China). Myocardial ischemia was established as previously described (22). Briefly, SD rats were anaesthetized by intraperitoneal injection of sodium pentobarbital ( $30 \mathrm{mg} / \mathrm{kg}$ body weight) and ventilated with oxygen using a small animal ventilator. After an incision in the left thorax at the level between the fourth and fifth ribs, the heart was exposed, and a 6-0 silk suture slipknot was placed around the proximal left anterior descending coronary artery (LAD) approximately $2 \mathrm{~mm}$ below the left auricle. Successful myocardial infarction injury was identified by the blanched appearance of the ligation region and marked arrhythmia. Sham-operated rats underwent the same surgical procedures except for the suture around the LAD which was not ligated. Experimental rats were randomly divided into 2 groups. Experimental rats were subjected to intragastric oral administration (p.o.) Tan-IIA $(10 \mathrm{mg} / \mathrm{kg} ; \mathrm{n}=10)$ or PBS $(\mathrm{n}=10)$. The treatment continued 10 times twice a day for a total 20-day therapeutic period. All the rats in the study were housed in an environment at $23 \pm 1.0^{\circ} \mathrm{C}$ and $50 \pm 5 \%$ humidity with $12-\mathrm{h}$ light/dark circadian cycle and ad libitum access to food and water. The mice were caged for $24 \mathrm{~h}$ since the last injection and the myocardial tissues were collected after cardiac perfusion. No rats succumbed during the experiments. On day 21 , experimental animals were euthanized under intravenous injection of pentobarbital $(40 \mathrm{mg} / \mathrm{kg})$, and efforts were made to minimize the suffering of the rats. Cervical dislocation was used as the euthanasia method. The myocardial tissues were used for immunohistochemical analysis.

Analysis of cardiac function and myocardial infarct size. Experimental rats were anesthetized with isoflurane and following procedures were processed according to a previous study (23). The parameters of left ventricular end-diastolic diameter (LVEDD), left ventricular end-systolic diameter (LVESD), left ventricular ejection fraction (LVEF) and left ventricular fractional shortening (LVES) were evaluated using the Sequoia 512 echocardiography system (Siemens Healthineers) following the manufacturer's instructions. The size of the myocardial infarction was assessed by 2,3,5-triphenyltetrazolium chloride (TTC) and Evan blue double staining assay as previously described (24). The infarct size in tissue sections was assessed by computerized planimetry and quantitated using ImageJ v2.0 (National Institutes of Health).

Immunohistochemical analysis. On day 21, myocardial tissues were obtained from experimental rats, immediately excised and placed in a $4 \%$ paraformaldehyde solution overnight at $4^{\circ} \mathrm{C}$, followed by dehydration, washing with PBS, and paraffin embedding. Paraffin-embedded myocardial tissues were cut into $4-\mu \mathrm{m}$ sections, subjected to hydrogen peroxide (3\%) for $10 \mathrm{~min}$, and blocked with BSA (5\%) for $2 \mathrm{~h}$ at $37^{\circ} \mathrm{C}$. Myocardial tissue sections were incubated with rabbit anti-rat antibodies Bcl-2 (1:1,000; product code ab182858), Bcl-xL (1:1,000; product code ab32370), caspase-3 (1:1,000; product code ab184787), cytoplasmic cytochrome $c$ (Cyto $c$; 1:1,000; product code ab133504), Apaf-1 (1:1,000; product code ab234436; all from Abcam) overnight at $4^{\circ} \mathrm{C}$. All sections were washed 3 times and incubated with horseradish peroxidase (HRP)-conjugated goat anti-rabbit IgG (1:2,000; product code ab205718; Abcam) for $1 \mathrm{~h}$ at $37^{\circ} \mathrm{C}$. The myocardial sections were stained with a 3,3-diaminobenzidine substrate system (Thermo Fisher Scientific, Inc.) for $1 \mathrm{~h}$ at room temperature. Images were captured using a light microscope (BX51; Olympus Corporation) under a magnification of x100.

Cells and reagents. Tan-IIA (purity $>99.2 \%$ ) was purchased from Herbasin (Shenyang) Co., Ltd.; Dasherb Corp. and dissolved in dimethyl sulfoxide (DMSO). Myocardiocytes were isolated from experimental rats with myocardial ischemia as previously described (25). Briefly, after dissection, heart tissues were washed, rinsed with HEPES-buffered saline solution and then incubated at $37^{\circ} \mathrm{C}$ for $2 \mathrm{~h}$ with HEPES-buffered saline 
solution containing $1.2 \mathrm{mg} / \mathrm{ml}$ pancreatin and $0.14 \mathrm{mg} / \mathrm{ml}$ collagenase (Gibco; Thermo Fisher Scientific, Inc.). After centrifugation $\left(2,000 \mathrm{x}\right.$ g for $10 \mathrm{~min}$ at $\left.4^{\circ} \mathrm{C}\right)$, cells were resuspended in Dulbecco's modified Eagle's medium/Ham's F-12 (DMEM/F12; Invitrogen; Thermo Fisher Scientific, Inc.) supplemented with 5\% fetal bovine serum(FBS; Sigma-Aldrich; Merck KGaA), $0.1 \mathrm{mM}$ ascorbate, insulin-transferring-sodium selenite media supplement (Sigma-Aldrich; Merck KGaA), $100 \mu \mathrm{g} / \mathrm{ml}$ streptomycin, $100 \mathrm{U} / \mathrm{ml}$ penicillin and $0.1 \mathrm{mM}$ bromodeoxyuridine. Cells were then diluted to $1 \times 10^{5}$ cells $/ \mathrm{ml}$ and cultured in DMEM/F12 supplemented with 10\% FBS. Myocardiocytes were treated with $\mathrm{H}_{2} \mathrm{O}_{2}(1 \mu \mathrm{M})$ and/or concentrations of Tan-IIA $(0-40 \mu \mathrm{M})$ and/or tunicamycin (TM; $1 \mu \mathrm{M}$; Sigma-Aldrich; Merck KGaA) for 24,48 and $72 \mathrm{~h}$ at $37^{\circ} \mathrm{C}$. PBS buffer containing 10\% DMSO (pH 7.2) was used as the control. All cells were maintained at $37^{\circ} \mathrm{C}$ in a humidified atmosphere containing $5 \% \mathrm{CO}_{2}$.

Counting Kit-8 (CCK-8) assay. The regulatory effects of Tan-IIA on proliferative ability of myocardiocytes were examined by CCK-8 assay as previously described (26). Briefly, the treated myocardiocytes were seeded into 96-well plates $\left(1 \times 10^{3}\right.$ cells/well $)$ and cultured at $37^{\circ} \mathrm{C}$ in a humidified incubator containing $5 \% \mathrm{CO}_{2}$. Then, $10 \mu \mathrm{l}$ of CCK-8 reagent (Beijing Solarbio Science \& Technology Co., Ltd.) was added to the cells followed by incubation at $37^{\circ} \mathrm{C}$ for $2 \mathrm{~h}$ according to the manufacturer's instructions. Cell viability was determined by a microplate reader (Eon BioTech, Pte Ltd.) at $450 \mathrm{~nm}$. Each experiment was repeated for 3 times.

Caspase-3 overexpression. The regulatory effects of caspase-3 overexpression on Tan-IIA-regulated apoptotic factors in myocardiocytes were examined by stable transfection. Expression plasmid pRK5-caspase-3 (casp-3OP) with a Flag tag (cat. no. PPL00180-2a; Public Protein/Plasmid Library) at the C-terminus was constructed by Invitrogen; Thermo Fisher Scientific, Inc.. Briefly, myocardiocytes $\left(1 \times 10^{5}\right.$ cells $\left./ \mathrm{ml}\right)$ were cultured at $37^{\circ} \mathrm{C}$ in $\mathrm{DMEM} / \mathrm{F} 12$ (Thermo Fisher Scientific, Inc.) supplemented with 10\% FBS (Sigma-Aldrich; Merck KGaA) in 6-well plates. After 24 h, myocardiocytes were transfected with plasmid containing either pRK5-caspase-3 $(0.5 \mu \mathrm{g})$ or pRK5-vector $(0.5 \mu \mathrm{g})$ by using Lipofectamine 2000 (Thermo Fisher Scientific, Inc.) at $37^{\circ} \mathrm{C}$ for $72 \mathrm{~h}$ according to the manufacturer's instructions. After $72 \mathrm{~h}$ of transfection, expression of caspase-3 was evaluated using western blot analysis and cells were used for further experiments.

TUNEL analysis. The terminal deoxynucleotidyl transferase (TdT)-mediated dUTP nick end labeling (TUNEL) assay (Roche Diagnostics $\mathrm{GmbH}$ ) was used to detect apoptosis in myocardial tissue and myocardiocytes. Briefly, heart tissues were immediately excised and placed in a $4 \%$ paraformaldehyde solution for $12 \mathrm{~h}$ at $4^{\circ} \mathrm{C}$. This was followed by dehydration, washing with PBS, and paraffin embedding. The paraffin blocks were cut into $4-\mu \mathrm{m}$ sections and stained with hematoxylin and eosin (H\&E; Beijing Solarbio Science \& Technology Co., Ltd.) for $15 \mathrm{~min}$ at room temperature according to the manufacturer's instructions. For myocardiocytes, the treated cells $\left(1 \times 10^{5}\right.$ cells) were cultured in 6 -well plates for $12 \mathrm{~h}$ at $37^{\circ} \mathrm{C}$, washed with PBS and fixed with $4 \%$ paraformaldehyde for $12 \mathrm{~h}$ at $4^{\circ} \mathrm{C}$. The cells were washed with PBS and stained using a TUNEL kit for $30 \mathrm{~min}$ at $25^{\circ} \mathrm{C}$ according to the manufacturer's protocols. Finally, the cells were counterstained with 5\% DAPI (Sigma-Aldrich; Merck KGaA) for $10 \mathrm{~min}$ at room temperature. Images from 6 randomly selected fields of view were captured using a fluorescence microscope at a magnification of x50 (DMI3000B; Leica Microsystems, Inc.).

Western blot analysis. The treated myocardiocytes were lysed using RIPA lysis buffer (Sigma-Aldrich; Merck KGaA). The cells were centrifuged at $12,000 \mathrm{x}$ g for $10 \mathrm{~min}$ at $4^{\circ} \mathrm{C}$. The concentration of protein was determined using a bicinchoninic acid assay (Thermo Fisher Scientific, Inc.). A total of $30 \mu \mathrm{g}$ of protein/lane was separated using 12\% SDS-PAGE, blocked with 5\% BSA (Sigma-Aldrich; Merck KGaA) overnight at $4^{\circ} \mathrm{C}$, and then transferred to a PVDF (EMD Millipore) membrane. Membranes were incubated with appropriate primary antibodies: Bcl-2 (1:1,000), Bak (1:1,000; product code ab32371; Abcam,), caspase-3 (1:1,000), Cyto $c(1: 1,000)$, Apaf-1 (1:1,000), Bim (1:1,000; product code ab32158; Abcam), CHOP (1:1,000; product code ab11419; Abcam) and $\beta$-actin (1:1,000; product code ab8227; Abcam) overnight at $4^{\circ} \mathrm{C}$, washed with PBS and incubated with horseradish peroxidase (HRP)-conjugated secondary antibodies (1:2,000, product code ab7090; Abcam) for $2 \mathrm{~h}$ at $25^{\circ} \mathrm{C}$. Protein expression was evaluated using enhanced chemiluminescence (product no. CPS1A300; Sigma-Aldrich; Merck KGaA). The expression of protein was quantified using LabWorks ${ }^{\mathrm{TM}}$ Image Acquisition (version 4.0; UVP, LLC).

Confocal laser microscopy. The treated myocardiocytes were cultured in 6-well plates for $12 \mathrm{~h}$ at $37^{\circ} \mathrm{C}$, washed with PBS, fixed with $4 \%$ paraformaldehyde for $15 \mathrm{~min}$ at room temperature, and then incubated with $0.1 \%$ Triton $\mathrm{X}-100$ and $1 \%$ BSA for $30 \mathrm{~min}$ at room temperature. Myocardiocytes were incubated with primary anti-mouse antibodies against Bim $(1: 1,000)$, CHOP $(1: 1,000)$, activating transcription factor 4 (ATF4; 1:1,000; product code ab216839; Abcam), inositol-requiring enzyme $1 \alpha$ (IRE1 $\alpha ; 1: 1,000$; product code ab37073; Abcam), thiobarbituric acid reactive substances (TBARS; 1:1,000; ab118970; Abcam), reactive oxygen species (ROS; 1:1,000; ab186027; Abcam), $\mathrm{H}_{2} \mathrm{O}_{2}$ (1:1,000; ab138874; Abcam) overnight at $4{ }^{\circ} \mathrm{C}$. Myocardiocytes were washed with PBS and incubated with corresponding anti-rabbit secondary antibody $(1: 2,000$, product code ab7090; Abcam) for $2 \mathrm{~h}$ at $25^{\circ} \mathrm{C}$. Subsequently, myocardiocytes were stained with $5 \%$ DAPI for $30 \mathrm{~min}$ at room temperature. Images of myocardiocytes were captured using a Zeiss confocal spectral microscope (Carl Zeiss AG) at a magnification of $\mathrm{x} 100$.

Statistical analysis. All data are expressed as the mean \pm SD of triplicate dependent experiments. All data were analyzed using SPSS Statistics 22.0 (IBM Corp.) Paired Student's test was used to assess the significant differences between two groups. One-way variance analysis (ANOVA) followed by Tukey's HSD test were used to assess the significant differences among multiple comparisons. $\mathrm{P}<0.05$ was considered to indicate a statistically significant difference. 
A

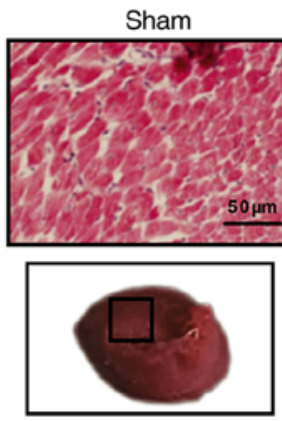

$\mathrm{B}$

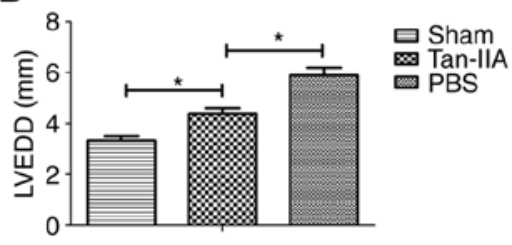

Tan-IIA
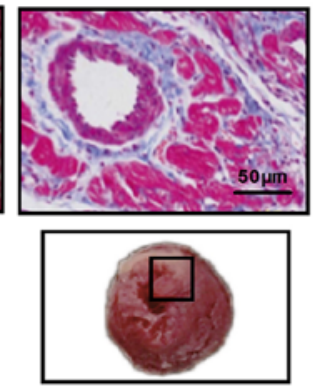

C
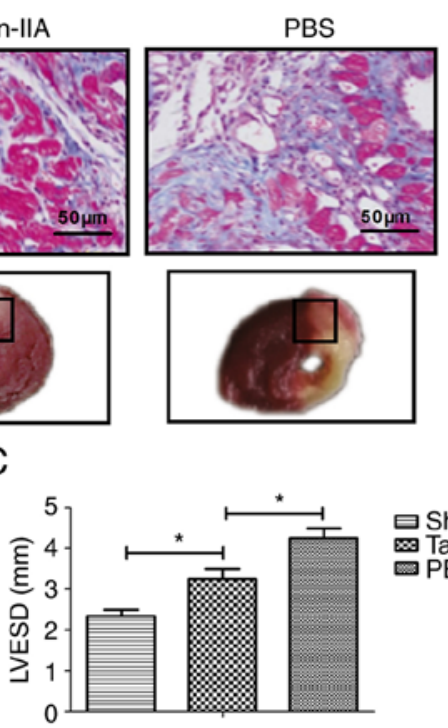

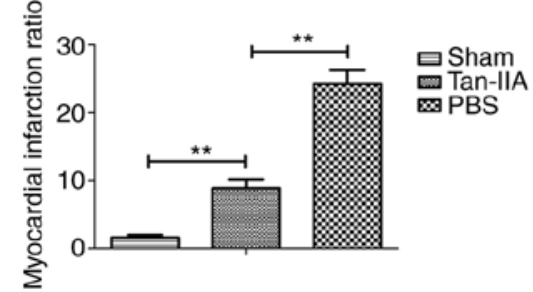

D

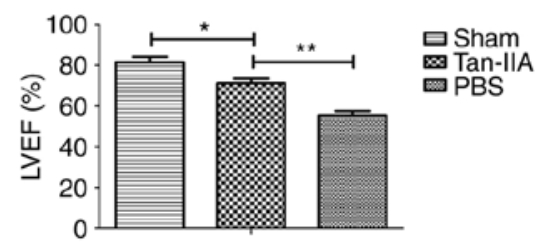

E

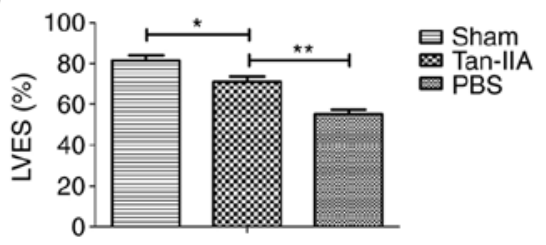

$\mathrm{F}$

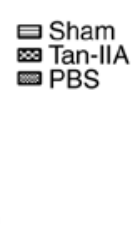

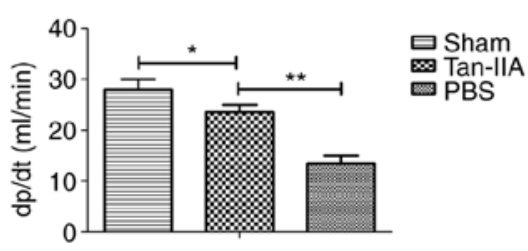

Figure 1. Therapeutic effect of Tan-IIA on a myocardial ischemia-reperfusion rat model. (A) TTC and Evan blue double staining for myocardial tissue and the myocardial infarct ratio of the PBS, Tan-IIA and sham groups. (B-E) Assessment of (B) LVEDD, (C) LVESD, (D) LVEF and (E) LVES in experimental rat PBS, Tan-IIA and sham groups. (F) Coronary flow in experimental rat PBS, Tan-IIA and sham groups. ${ }^{*} \mathrm{P}<0.05,{ }^{* *} \mathrm{P}<0.01$. $\mathrm{n}=6$ animals in each group. The results are expressed as the mean \pm SD. Tan-IIA, tanshinone-IIA; TTC, 2,3,5-triphenyltetrazolium chloride; LVEDD, left ventricular end-diastolic diameter; LVESD, left ventricular end-systolic diameter; LVEF, left ventricular ejection fraction; LVES, left ventricular fractional shortening.

\section{Results}

Protective effect of Tan-IIA in myocardial ischemiareperfusion rat model. To determine the therapeutic effect of Tan-IIA in myocardial ischemia, a myocardial ischemia-reperfusion rat model was established and received treatment with Tan-IIA, or PBS. As demonstrated in Fig. 1A, Tan-IIA attenuated the myocardial infarction sizes after ischemia-reperfusion compared with the PBS group $(\mathrm{P}<0.01)$. In addition, the myocardial functions LVEDD, LVESD, LVEF and LVES were significantly ameliorated during reperfusion in the Tan-IIA group compared with the PBS group (Fig. 1B-E). Tan-IIA treatment exhibited a significant elevation in $\mathrm{dp} / \mathrm{dt}(\max )$ throughout the reperfusion period compared with the PBS $(\mathrm{P}<0.01$; Fig. 1F). These data indicated that Tan-IIA played a protective role in a myocardial ischemia-reperfusion rat model.

Tan-IIA treatment inhibits myocardiocyte apoptosis in a rat model of myocardial ischemia. The anti-apoptotic role of Tan-IIA in a rat model of myocardial ischemia was next analyzed. A TUNEL assay demonstrated that Tan-IIA decreased apoptosis of myocardial tissue compared with PBS (Fig. 2A). Immunohistochemical analysis revealed that Tan-IIA upregulated the anti-apoptotic protein Bcl-2 and Bcl-xL expression in myocardial tissue compared with PBS (Fig. 2B). The data also demonstrated that Tan-IIA downregulated pro-apoptotic protein caspase-3, Cyto $c$ and
Apaf-1 expression in myocardial tissue compared with PBS (Fig. 2C). These data indicated that Tan-IIA could inhibit apoptosis of myocardial tissue in rat model of myocardial ischemia.

Effect of Tan-IIA on protection in $\mathrm{H}_{2} \mathrm{O}_{2}$-induced myocardiocytes. To verify the protective effect of Tan-IIA on myocardiocytes, viability of myocardiocytes was analyzed in vitro. As demonstrated in Fig. 3A, $30 \mu \mathrm{M}$ of Tan-IIA presented the optimal protective effect on viability of myocardiocytes. As demonstrated in Fig. 3B, Tan-IIA $(30 \mu \mathrm{M})$ increased the viability of myocardiocytes in a time-dependent manner compared with PBS. These data indicated that Tan-IIA could increase the viability of myocardiocytes.

Tan-IIA treatment inhibits myocardiocyte apoptosis in myocardiocytes in vitro. The anti-apoptotic effect of Tan-IIA was analyzed in myocardiocytes in vitro. As revealed in Fig. 4A, Tan-IIA produced a significant reduction of apoptosis of myocardiocytes compared with PBS treatment. The results revealed that expression levels of Bcl-2 and Bcl-xl were upregulated by treatment with Tan-IIA in myocardiocytes compared with PBS treatment (Fig. 4B). Western blot analysis demonstrated that apoptotic factors including cleaved caspase-3 (casp-3), Cyto $c$ and Apaf-1 in the mitochondrial apoptotic pathway were downregulated by Tan-IIA in myocardiocytes compared with treatment by 
A
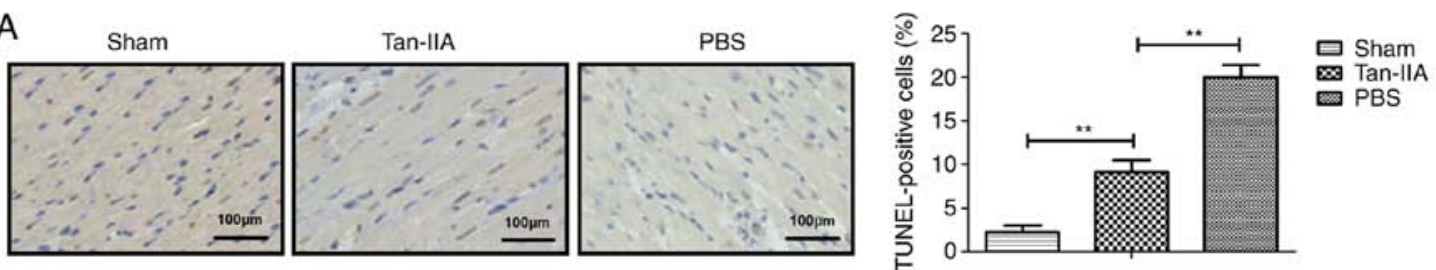

B

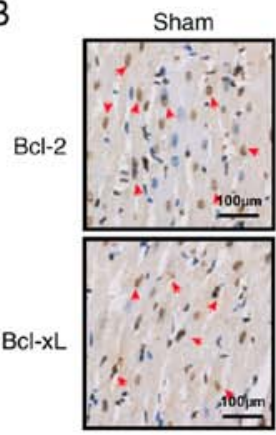

Tan-IIA

PBS
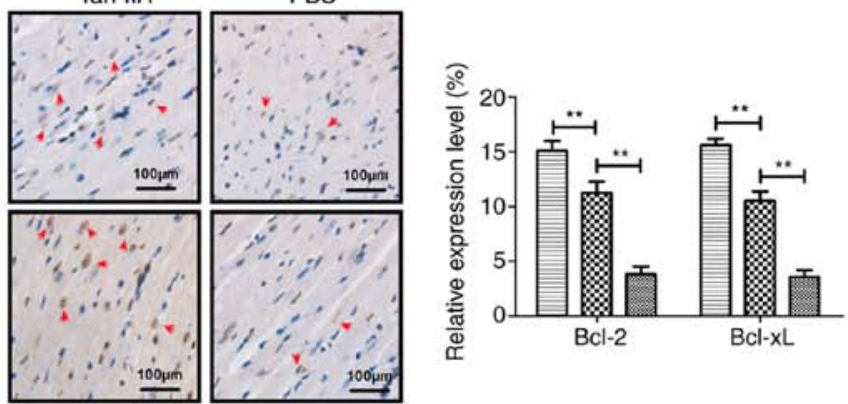

Sham

Tan-I

C
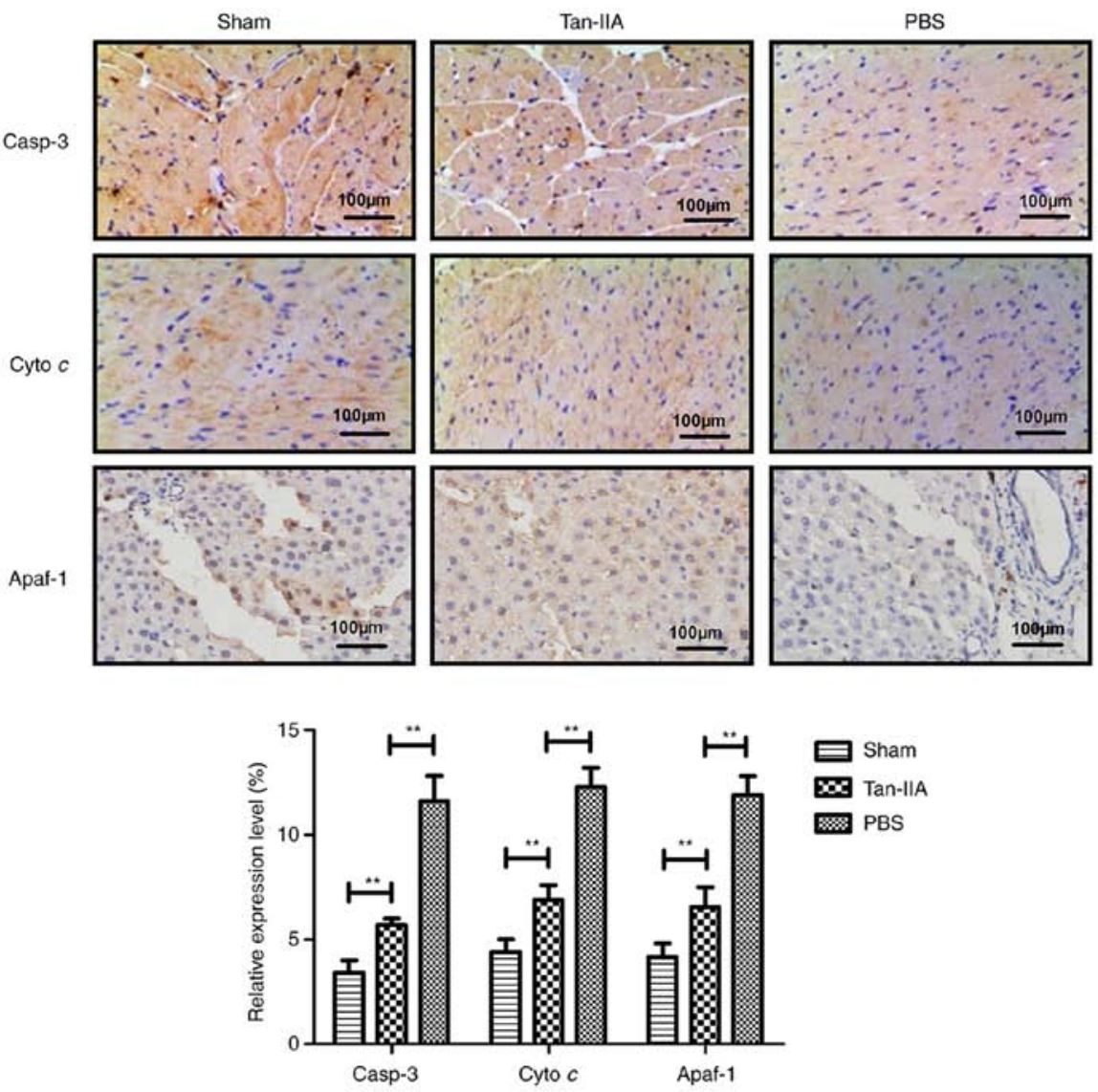

Figure 2. Tan-IIA decreases apoptosis of myocardial tissue in a rat model of myocardial ischemia. (A) Apoptotic myocardiocytes in myocardial tissue of sham, Tan-IIA and PBS groups. (B) Anti-apoptotic protein Bcl-2 and Bcl-xL expression in myocardial tissue determined by immunohistochemistry. (C) Pro-apoptotic protein caspase-3, Cyto $c$ and Apaf-1 expression in myocardial tissue in experimental rat PBS, Tan-IIA and sham groups. ${ }^{* *}<0.01$. Tan-IIA, tanshinone-IIA; Cyto $c$, cytochrome $c$.

PBS (Fig. 4C). These data indicated that Tan-IIA may inhibit myocardiocyte apoptosis via the mitochondrial apoptotic pathway.

Tan-IIA improves myocardial ischemia by regulating oxidative stress. The effect of Tan-IIA on oxidative stress was analyzed in myocardiocytes. As demonstrated in Fig. 5A and B, Tan-IIA treatment upregulated the expression of endoplasmic reticulum stress-related proteins Bim and CHOP in myocardiocytes compared with the control group. However, the oxidative stress stimulator TM abolished the Tan-IIA-increased Bim and CHOP expression in myocardiocytes compared with the control group. The data also demonstrated that myocardial injury-induced apoptosis was inhibited by Tan-IIA pretreatment compared with the control group and this effect was abolished by TM treatment in myocardiocytes (Fig. 5C). These 


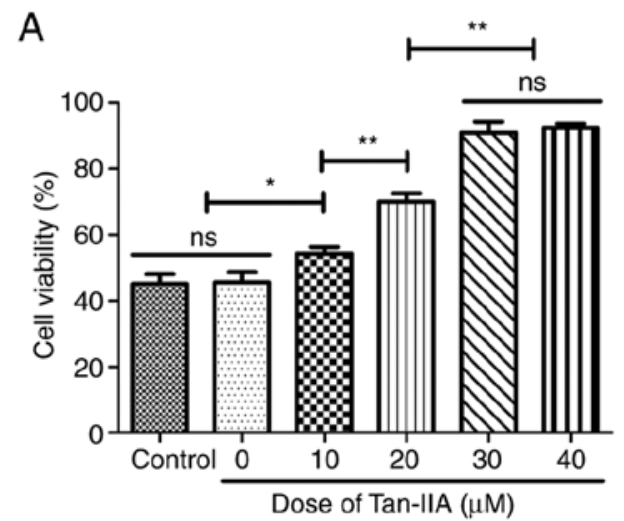

B

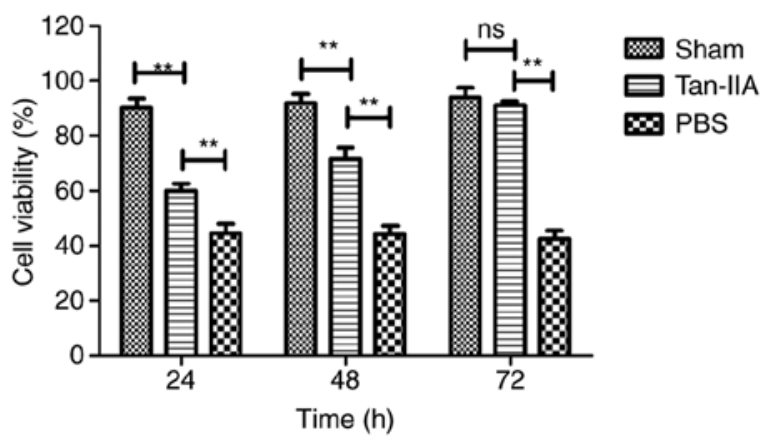

Figure 3. Effect of Tan-IIA on viability of $\mathrm{H}_{2} \mathrm{O}_{2}$-induced myocardiocytes. (A) Viability of $\mathrm{H}_{2} \mathrm{O}_{2}$-induced myocardiocytes after treatment with Tan-IIA $(0-40 \mu \mathrm{M})$. (B) Effect of $30 \mu \mathrm{M}$ of Tan-IIA on viability of $\mathrm{H}_{2} \mathrm{O}_{2}$-induced myocardiocytes at the indicated time-points $(24,48$ and $72 \mathrm{~h}) .{ }^{* *} \mathrm{P}<0.05$ and ${ }^{* * *} \mathrm{P}<0.01$. Tan-IIA, tanshinone-IIA; ns, no significance.

A

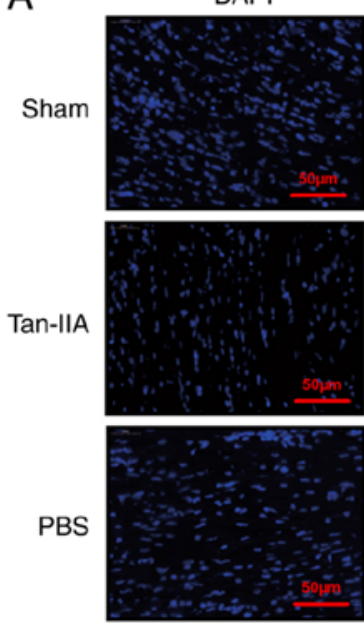

B

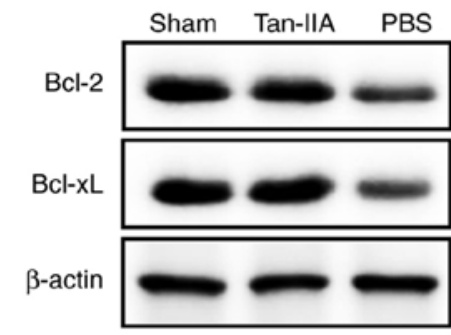

Merge
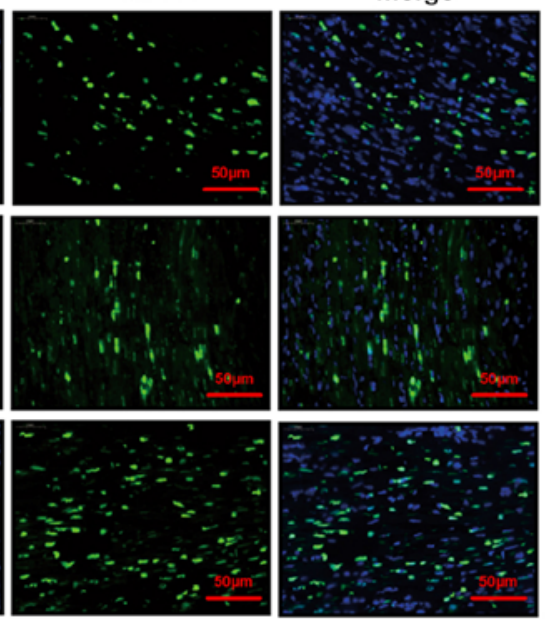
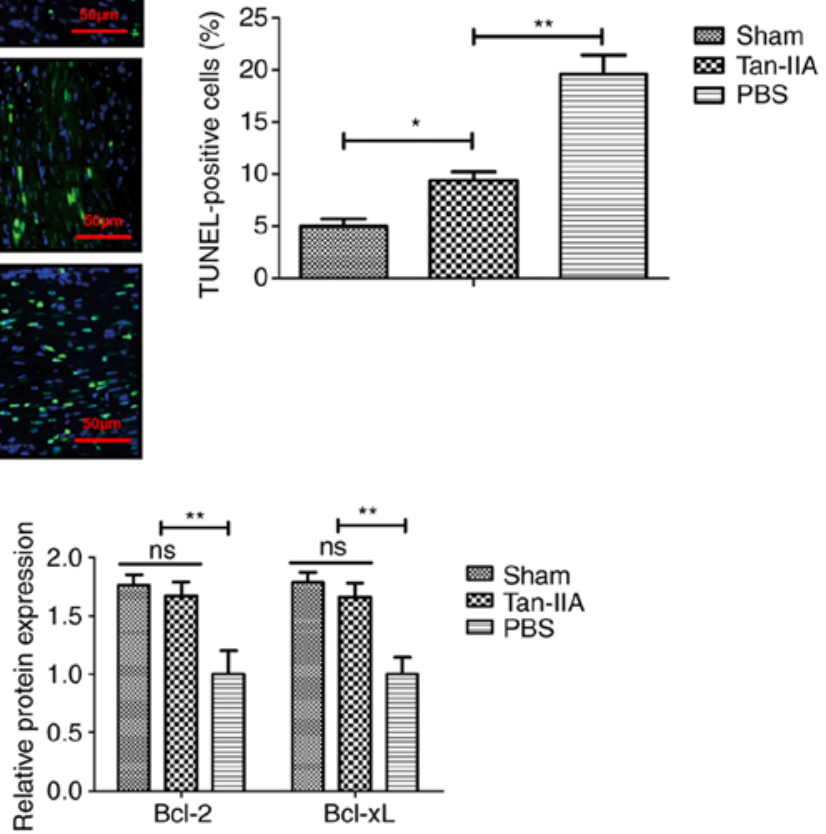

C Sham Tan-IIA PBS
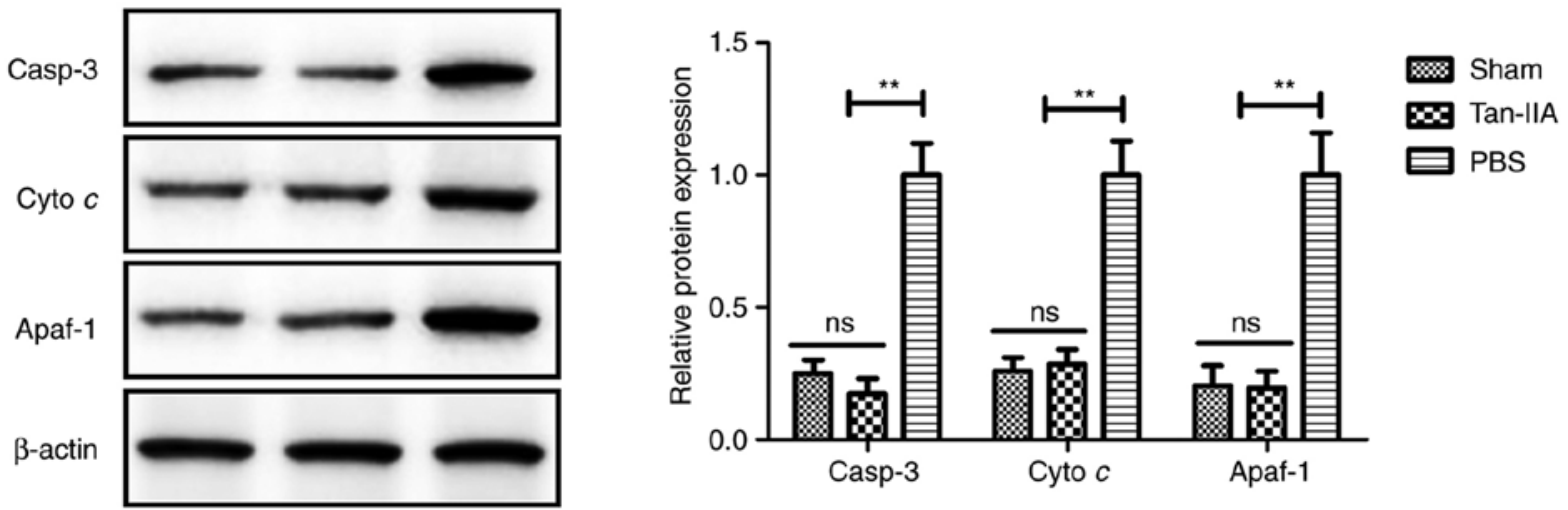

Figure 4. Tan-IIA inhibits myocardiocyte apoptosis. (A) In vitro effect of Tan-IIA on apoptosis of myocardiocytes induced by $\mathrm{H}_{2} \mathrm{O}_{2}$. (B) Protein expression level of Bcl-2 and Bcl-xL in myocardiocytes among Tan-IIA, Sham and PBS groups. (C) Protein expression level of casp-3, Cyto $c$, and Apaf-1 in myocardiocytes among Tan-IIA, Sham and PBS groups. ${ }^{*} \mathrm{P}<0.05$ and ${ }^{* *} \mathrm{P}<0.01$. Tan-IIA, tanshinone-IIA; Cyto $c$, cytochrome $c$; ns, no significance. 
A

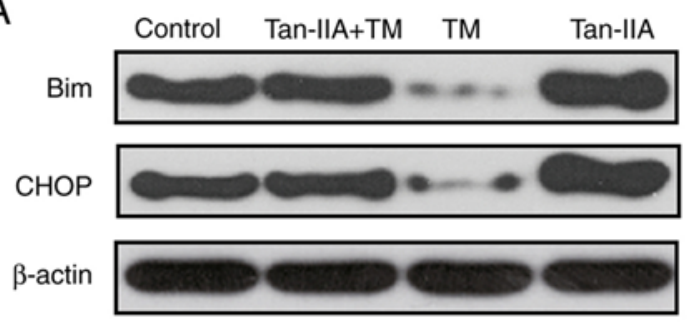

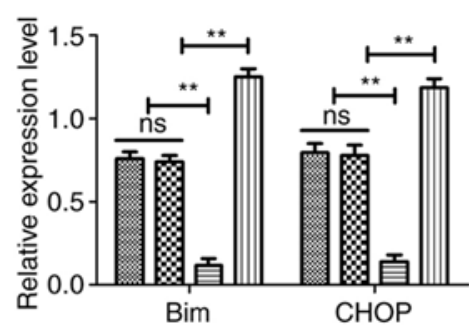

Control

$\infty$ Tan-IIA+TM

曰 $\mathrm{TM}$

띠 Tan-IIA

B
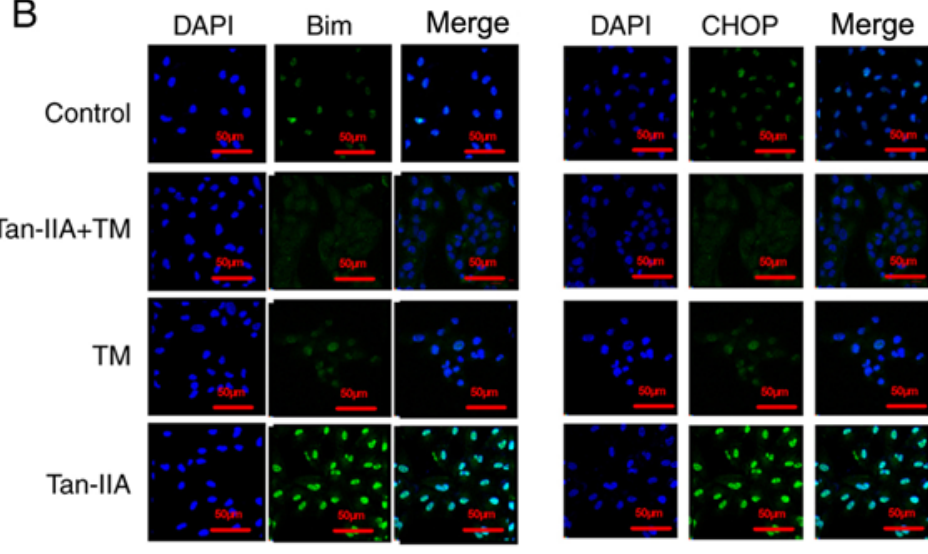

C
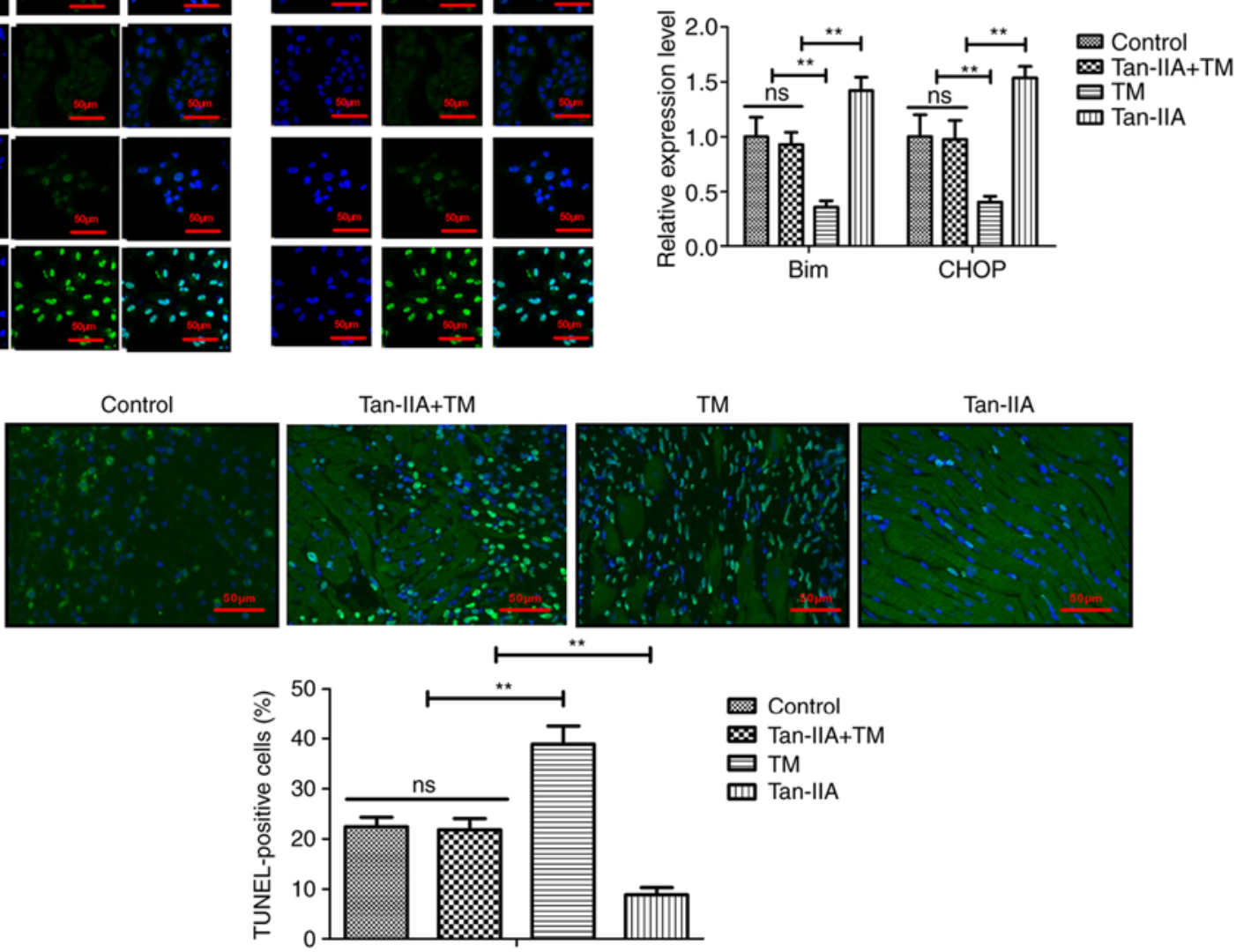

DAPI/TUNEL

Figure 5. Tan-IIA decreases the endoplasmic reticulum stress-dependent pathway in $\mathrm{H}_{2} \mathrm{O}_{2}$-induced myocardiocytes. (A) Bim and CHOP expression in myocardiocytes determined by western blot analysis. (B) Bim and CHOP expression in myocardiocytes determined by immunofluorescence. (C) Effect of TM on Tan-IIA-decreased apoptosis of myocardiocytes determined by TUNEL assay. ${ }^{* *} \mathrm{P}<0.01$. Tan-IIA, tanshinone-IIA; TM, tunicamycin; TUNEL, terminal deoxynucleotidyl transferase (TdT)-mediated dUTP nick end labeling; ns, no significance.

results indicated that Tan-IIA could protect myocardiocytes against apoptosis by modulating oxidative stress.

Tan-IIA improves myocardial ischemia by decreasing apoptosis of myocardiocytes via inhibition of the mitochondrial apoptotic signaling pathway. To identify the possible mechanism of Tan-IIA in myocardiocytes, caspase-3-overexpressed (casp-3OP) myocardiocytes were established. As demonstrated in Fig. 6A, caspase-3 overexpression reversed Tan-IIA-decreased Casp-3, Cyto $c$, and Apaf-1 in myocardiocytes. The results in Fig. 6B demonstrated that Casp-3 overexpression reversed Tan-IIA-decreased apoptosis of myocardiocytes compared with the control group. These results indicated that Tan-IIA could decrease apoptosis of myocardiocytes via inhibition of the mitochondrial apoptotic signaling pathway.
Effects of Tan-IIA on intracellular calcium and oxidative stress in myocardiocytes. Previous data has revealed that the intracellular calcium level and oxidative stress are increased during apoptosis of myocardiocytes (21). It has been demonstrated that $\mathrm{I} / \mathrm{R}$ injury induces oxidative and inflammatory responses, and further ultimately damages cardiac function in patients suffering myocardial ischemia (27). To investigate whether the protective effect of Tan-IIA on myocardiocytes in myocardial ischemia rats is related with intracellular calcium and oxidative stress, the intracellular calcium and oxidative stress levels were analyzed. As demonstrated in Fig. 7A, Tan-IIA decreased the level of intracellular calcium in myocardiocytes compared with the control. The results revealed that production of TBARS, ROS and $\mathrm{H}_{2} \mathrm{O}_{2}$ was inhibited by Tan-IIA compared with the control groups (Fig. 7B-D). The data also demonstrated that Tan-IIA decreased 

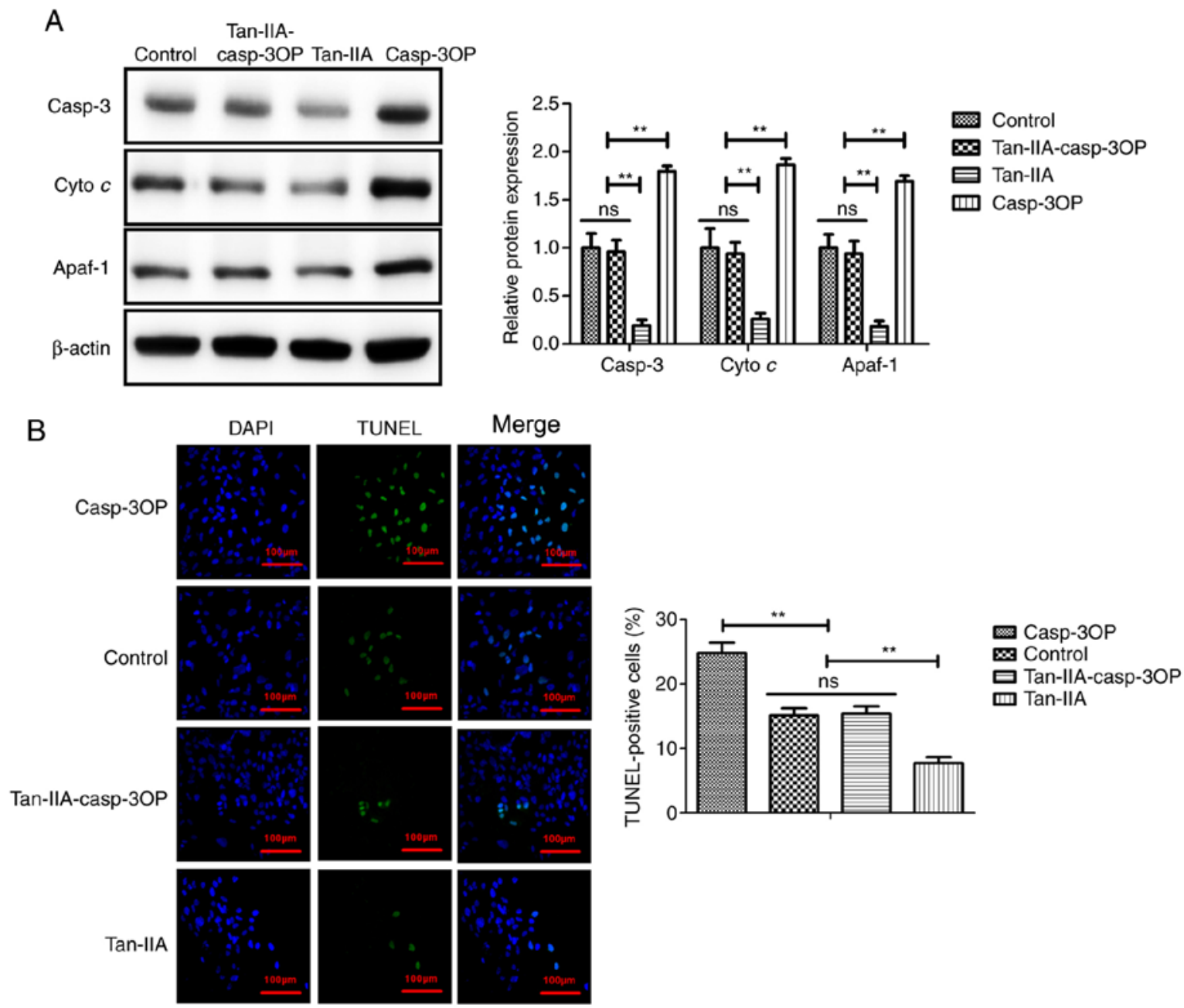

Figure 6. Effects of Tan-IIA on the mitochondrial apoptotic signaling pathway in $\mathrm{H}_{2} \mathrm{O}_{2}$-induced apoptosis in myocardiocytes. (A) Effect of casp-3OP on Tan-IIA-regulated Casp-3, Cyto $c$, and Apaf-1 expression in myocardiocytes determined by western blot analysis. (B) Effect of casp-3OP on Tan-IIA-decreased apoptosis of myocardiocytes determined by TUNEL assay. ${ }^{*} \mathrm{P}<0.01$. Tan-IIA, tanshinone-IIA; casp-3OP, caspase-3-overexpressed; Cyto $c$, cytochrome $c$; TUNEL, terminal deoxynucleotidyl transferase (TdT)-mediated dUTP nick end labeling; ns, no significance.

expression of ATF4 and IRE1 $\alpha$ expression in myocardiocytes (Fig. 7E and F). Collectively, these results indicated that Tan-IIA could decrease intracellular calcium and oxidative stress in myocardiocytes.

\section{Discussion}

Tan-IIA is an effective drug for the treatment of cardiovascular diseases (28). A previous study revealed that Tan-IIA injection was effective and safe in improving clinical outcomes in patients with coronary heart disease (15). Tan-IIA-decreased apoptosis of myocardiocytes has been revealed to contribute to the recovery of myocardial function (17). However, the idiographic mechanism of Tan-IIA remains unknown in myocardiocytes. The therapeutic effect of Tan-IIA on inhibition of myocardial tissue apoptosis in an experimental rat model of myocardial ischemia and the possible mechanism of Tan-IIA in myocardiocytes were investigated in the present study. The results provided insights on Tan-IIA-induced cellular mechanisms for anti-apoptotic activities in impaired myocardiocytes undergoing the oxidative stress-dependent pathway and mitochondrial signaling pathway, which demonstrated the potential value of using Tan-IIA for cardiovascular disease therapy.

Apoptosis of myocardiocytes has been revealed to be induced by myocardial ischemia caused by hypoxia, while reperfusion aggravates the apoptotic process during the preceding ischemic period (29). A previous study revealed that apoptosis of myocardiocytes leads to increasing intracellular calcium and oxidative stress, which aggravates the inflammatory response and activation of proapoptotic signaling proteins during the reperfusion period (30). Our results demonstrated that Tan-IIA improved myocardial infarction size, myocardial functions, such as dp/dt, coronary flow and LVDP. The data also revealed that Tan-IIA could inhibit apoptosis of myocardial tissue in a rat model of myocardial ischemia, indicating that Tan-IIA has clinical value.

A previous study reported that apoptosis mediated by endoplasmic reticulum stress partly depended on signaling through activation of PERK and EIF2 $\alpha$ expression (31). The data in this previous study identified that Tan-IIA decreased PERK and EIF2 $\alpha$ expression in myocardiocytes, which relieved endoplasmic reticulum stress and further led to reduction of apoptosis of myocardiocytes. In addition, intracellular calcium dynamics have been demonstrated to be important in promoting 

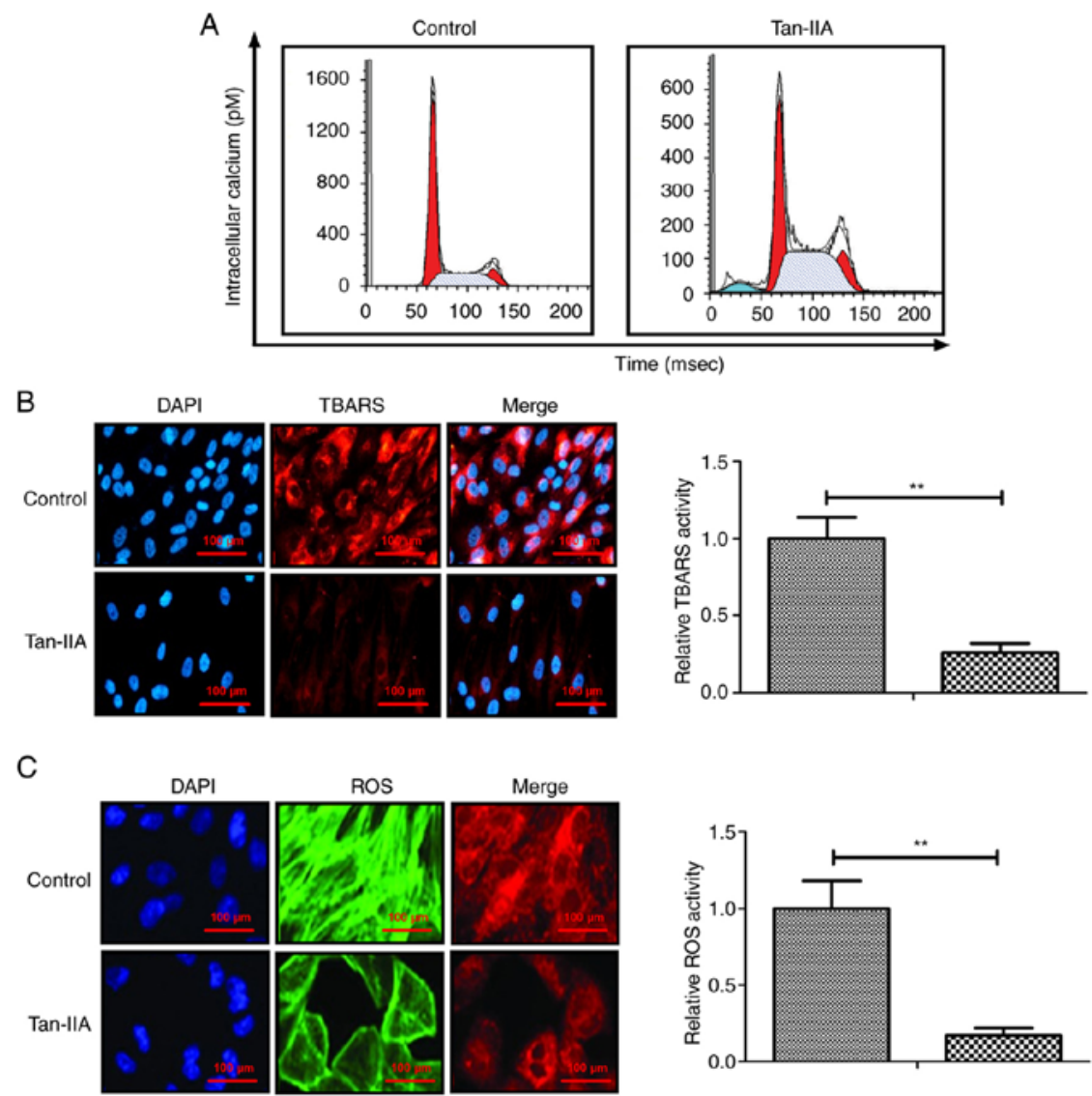

Control
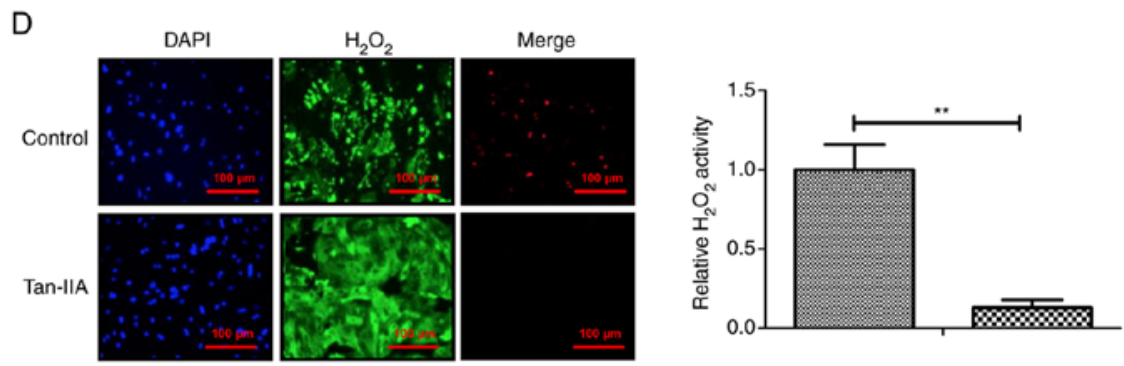

Control
Tan-IIA
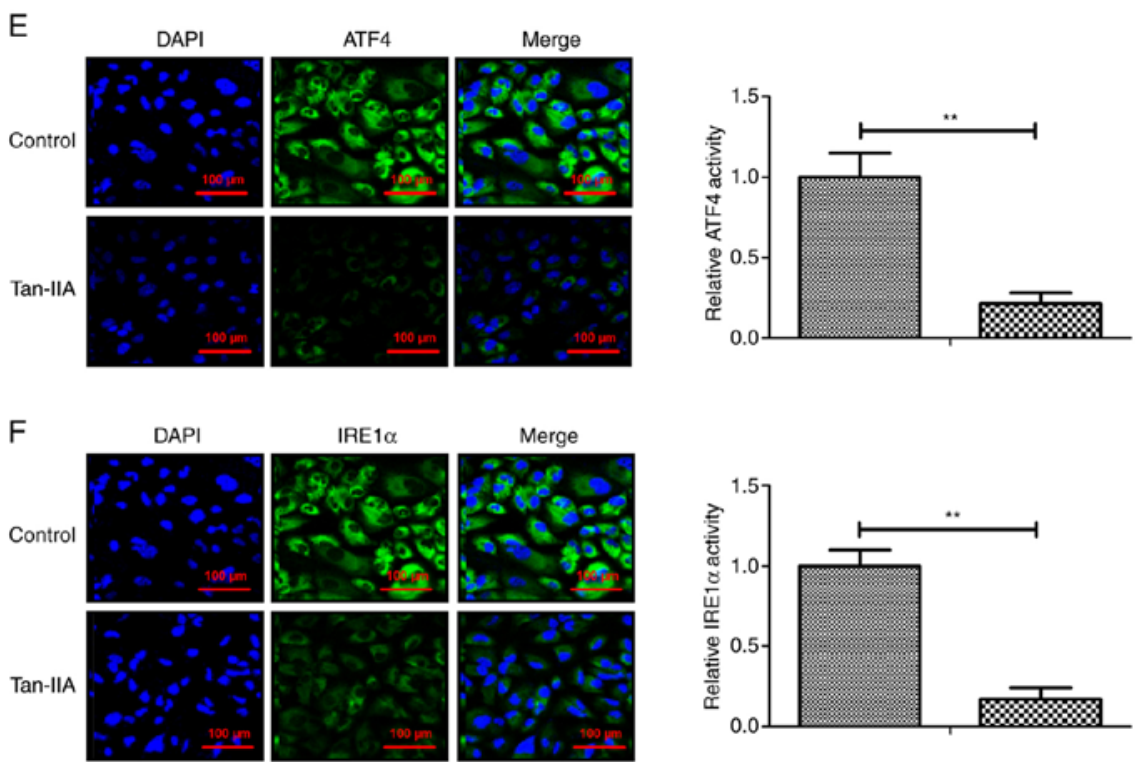

IRE1 $\alpha$

Merge
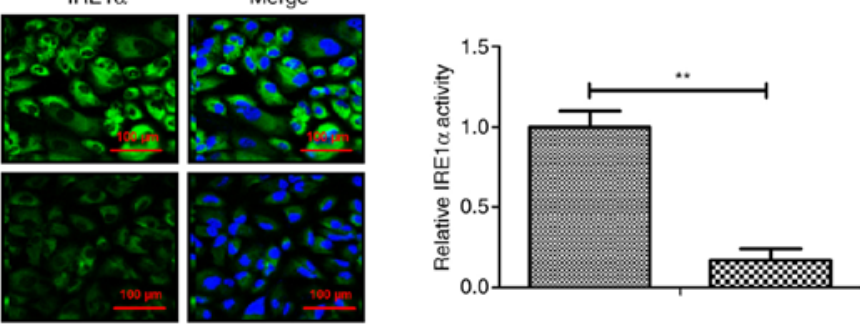

Contro

$\mathbb{1}$ Tan-IIA

$\notin$ Tan-IIA

$\infty$ Tan-IIA

Figure 7. Effects of Tan-IIA on intracellular calcium and oxidative stress in $\mathrm{H}_{2} \mathrm{O}_{2}$-induced myocardiocytes. (A) Effect of Tan-IIA on intracellular calcium in $\mathrm{H}_{2} \mathrm{O}_{2}$-induced myocardiocytes. (B-D) Effect of Tan-IIA on production of (B) TBARS, (C) ROS and (D) $\mathrm{H}_{2} \mathrm{O}_{2}$ in $\mathrm{H}_{2} \mathrm{O}_{2}$-induced myocardiocytes. (E and F) Effect of Tan-IIA on ATF4 and IRE1 $\alpha$ expression in myocardiocytes. ${ }^{* *} \mathrm{P}<0.01$. Tan-IIA, tanshinone-IIA; TBARS, thiobarbituric acid reactive substances; ROS, reactive oxygen species; ATF4, activating transcription factor 4 ; IRE1 $\alpha$, inositol-requiring enzyme $1 \alpha$. 
triggered activity during acetylcholine infusion in patients with heart failure (32). Furthermore, maintaining the balance between intracellular oxidants and antioxidants has been revealed to have a protective effect against myocardial (I/R) injury (33). The results of the present study revealed that Tan-IIA decreased intracellular calcium and downregulated TBARS, ROS and $\mathrm{H}_{2} \mathrm{O}_{2}$ production in myocardiocytes. Importantly, no side effects were observed with Tan-IIA treatment such as thrombotic phenomena, ulcerating atherosclerotic lesion or micronecrosis in experimental animals during experimentation.

Tan-IIA has cardioprotective function through multiple targets related with NO production, such as eNOS phosphorylation, L-arginine uptake and CAT expression, which may have major clinical implications (34). Inhibition of the mitochondrial apoptotic signaling pathway has been revealed to contribute to the improvement of cardiac function and energy metabolism in mice after myocardial ischemia injury (35). The present study revealed that Tan-IIA downregulated protein expression levels, such as cleaved caspase-3, Cyto $c$ and Apaf-1 in the mitochondria-mediated internal signaling pathway. A previous study reported that upregulation of Bcl-2 and Bak expression could inhibit apoptosis of cells during myocardial I/R injury (36). Although Bim protein is considered to localize in mitochondria and acts as an anti-apoptosis protein, it is also found on the endoplasmic reticulum and nuclear membranes (37). Additionally, Bak is downregulated during ischemia and/or reperfusion injury in myocardial infarction (38). In the present study, the results revealed that $\mathrm{Bcl}-2$ and Bak expression levels were upregulated in myocardiocytes, which were associated with the inhibition of the mitochondrial apoptotic signaling pathway during myocardial ischemia injury. The data revealed that Tan-IIA decreased apoptosis of myocardiocytes in a rat myocardial injury model and this was mediated by the mitochondrial signaling pathway. Wang et al reported that Bim is involved in protection of myocardial apoptosis after I/R injury (39). In the present study, our in vivo and in vitro data demonstrated that Tan-IIA not only increased Bim expression in myocardial tissue, but also upregulated Bim expression in myocardial cells. Yu et al reported that naringenin treatment protects against myocardial I/R injury by reducing oxidative stress and $\mathrm{CHOP}$ expression (40). The present study is the first, to the best of our knowledge, to elucidate the relationship between Tan-IIA and CHOP during ischemic heart disease. Collectively, Tan-IIA treatment may lead to the inhibition of downstream caspase-3 in apoptosis in myocardiocytes, which may be a potential drug for the treatment of patients with myocardial ischemia.

Certain limitations of the present study should be noted. Firstly, this study did not analyze the endoplasmic reticulum stress-dependent pathway. Notably, the main mechanism of Tan-IIA in myocardiocytes is intricate. Nevertheless, further experiments and data analysis should be conducted in future studies. Secondly, the effects of Tan-IIA on loss of the mitochondrial membrane potential and the content of Cyto $c$ in mitochondria and the cytoplasm in myocardiocytes were not analyzed. Thirdly, the specific anti-apoptotic pathway by which Tan-IIA protected against myocardial infarction was not confirmed. Finally, the sample size was small, therefore, the effect of Tan-IIA on myocardial infarct and apoptosis of myocardiocytes should be identified in a larger sample size to draw conclusions in our future study.
In conclusion, data in the present study indicated the therapeutic effect in a rat model of myocardial ischemia and provided a possible mechanism responsible for the anti-apoptotic effect of Tan-IIA in myocardiocytes. Our analysis revealed that Tan-IIA ameliorated apoptosis of myocardiocytes through the mitochondrial signaling pathway and improved myocardial function via oxidative stress-dependent pathways. The present study further demonstrated the anti-apoptotic effects of Tan-IIA in infarct expansion after myocardial ischemia, which provides a significant clinical reference for the treatment of patients with myocardial ischemia.

\section{Acknowledgements}

Not applicable.

\section{Funding}

The present study was supported by Medical Science and Technology Research Fund of Guangdong Province (grant no. A2019427) and Science and Technology Innovation Bureau of Shenzhen Nanshan District (grant no. 20199).

\section{Availability of data and materials}

All datasets generated or analyzed during the present study are available from the corresponding author upon reasonable request.

\section{Authors' contributions}

YF, CD, SC, ZL and WA collected and interpreted the data and wrote the manuscript. LW, PX, BJ and YF designed and performed the experiments. HF conceived the study, reviewed and edited the manuscript. All authors read and approved the final manuscript.

\section{Ethics approval and consent to participate}

The present study was approved (approval no. 20160512C10) by the Ethics Committee of Shenzhen Nanshan People's Hospital (Shenzhen, China).

\section{Patient consent for publication}

Not applicable.

\section{Competing interests}

The authors declare that they have no competing interests.

\section{References}

1. Dong Y, Chen H, Gao J, Liu Y, Li J and Wang J: Molecular machinery and interplay of apoptosis and autophagy in coronary heart disease. J Mol Cell Cardiol 136: 27-41, 2019.

2. Yndestad A, Sandanger $\varnothing$, Jong WMC, Aukrust P and Zuurbier CJ: Response to letter from Toldo et al on 'NLRP3 inflammasome activation during myocardial ischemia reperfusion is cardioprotective'. Biochem Biophys Res Commun 474: 328-329, 2016.

3. Wang Z, Zhang J, Ren T and Dong Z: Targeted metabolomic profiling of cardioprotective effect of Ginkgo biloba L. extract on myocardial ischemia in rats. Phytomedicine 23: 621-631, 2016. 
4. Wang L, Niu X, Hu J, Xing H, Sun M, Wang J, Jian Q and Yang H: After myocardial ischemia-reperfusion, miR-29a, and Let7 could affect apoptosis through regulating IGF-1. Biomed Res Int 2015: 245412, 2015.

5. Wakiyama H, Cowan DB, Toyoda Y,Federman M,Levitsky S and McCully JD: Selective opening of mitochondrial ATP-sensitive potassium channels during surgically induced myocardial ischemia decreases necrosis and apoptosis. Eur J Cardiothorac Surg 21: 424-433, 2002.

6. Elsässer A, Suzuki K, Lorenz-Meyer S, Bode C and Schaper J: The role of apoptosis in myocardial ischemia: A critical appraisal. Basic Res Cardiol 96: 219-226, 2001.

7. Niermann C, Gorressen S, Klier M, Gowert NS, Billuart P, Kelm M, Merx MW and Elvers M: Oligophrenin1 protects mice against myocardial ischemia and reperfusion injury by modulating inflammation and myocardial apoptosis. Cell Signal 28: 967-978, 2016

8. Guo CX, Jiang X, Zeng XJ, Wang HX, Li HH, Du FH and Chen BX: Soluble receptor for advanced glycation end-products protects against ischemia/reperfusion-induced myocardial apoptosis via regulating the ubiquitin proteasome system. Free Radic Biol Med 94: 17-26, 2016.

9. Song T, Yao Y, Wang T, Huang $\mathrm{H}$ and Xia H: Tanshinone IIA ameliorates apoptosis of myocardiocytes by up-regulation of miR-133 and suppression of caspase-9. Eur J Pharmacol 815: 343-350, 2017.

10. Inserte J, Cardona M, Poncelas-Nozal M, Hernando V Vilardosa Ú, Aluja D, Parra VM, Sanchis D and Garcia-Dorado D Studies on the role of apoptosis after transient myocardial ischemia: Genetic deletion of the executioner caspases-3 and -7 does not limit infarct size and ventricular remodeling. Basic Res Cardiol 111: 18, 2016

11. Dongó E, Hornyák I, Benko Z and Kiss L: The cardioprotective potential of hydrogen sulfide in myocardial ischemia/reperfusion injury (review). Acta Physiol Hung 98: 369-381, 2011.

12. Gao S, Liu Z,Li H,Little PJ, Liu P and Xu S: Cardiovascular actions and therapeutic potential of tanshinone IIA. Atherosclerosis 220: 3-10, 2012.

13. Mao C, Zhang Y, Zhang Y, Cao L, Shao H, Wang L, Zhu L and $\mathrm{Xu} Z$ : The effect of tanshinone IIA on the cardiovascular system in ovine fetus in utero. Am J Chin Med 37: 1031-1044, 2009.

14. Feng J, Li SS and Liang QS: Effects of Tanshinone II A on the myocardial apoptosis and the miR-133 levels in rats with heart failure. Zhongguo Zhong Xi Yi Jie He Za Zhi 32: 930-933, 2012 (In Chinese).

15. Yu ML, Li SM, Gao X, Li JG, Xu H and Chen KJ: Sodium tanshinone II A sulfonate for coronary heart disease: A systematic review of randomized controlled trials. Chin J Integr Med 26 219-226, 2020

16. Yang R, Liu A, Ma X, Li L, Su D and Liu J: Sodium tanshinone IIA sulfonate protects cardiomyocytes against oxidative stress-mediated apoptosis through inhibiting JNK activation. J Cardiovasc Pharmacol 51: 396-401, 2008.

17. Gao S, Li L, Li L, Ni J, Guo R, Mao J and Fan G: Effects of the combination of tanshinone IIA and puerarin on cardiac function and inflammatory response in myocardial ischemia mice. $\mathrm{J} \mathrm{Mol}$ Cell Cardiol 137: 59-70, 2019.

18. Aimo A, Castiglione V, Borrelli C, Saccaro LF, Franzini M, Masi S, Emdin M and Giannoni A: Oxidative stress and inflammation in the evolution of heart failure: From pathophysiology to therapeutic strategies. Eur J Prev Cardiol 27: 494-510, 2020.

19. van der Pol A, Gil A, Tromp J, Silljé HHW, van Veldhuisen DJ, Voors AA, Hoendermis ES, Grote Beverborg N, Schouten EM, de Boer RA, et al: OPLAH ablation leads to accumulation of 5-oxoproline, oxidative stress, fibrosis, and elevated fillings pressures: A murine model for heart failure with a preserved ejection fraction. Cardiovasc Res 114: 1871-1882, 2018.

20. Chang JP, Chen MC, Liu WH, Lin YS, Huang YK, Pan KL, Ho WC, Fang CY, Chen CJ and Chen HC: Mitochondrial apoptotic pathway activation in the atria of heart failure patients due to mitral and tricuspid regurgitation. Exp Mol Pathol 99: 65-73, 2015.

21. Dalal S, Zha Q, Singh M and Singh K: Osteopontin-stimulated apoptosis in cardiac myocytes involves oxidative stress and mitochondrial death pathway: Role of a pro-apoptotic protein BIK. Mol Cell Biochem 418: 1-11, 2016.

22. Kuznetsov G, Bush KT, Zhang PL and Nigam SK: Perturbations in maturation of secretory proteins and their association with endoplasmic reticulum chaperones in a cell culture model for epithelial ischemia. Proc Natl Acad Sci USA 93: 8584-8589, 1996

23. Zhang HZ, Kim MH, Lim JH and Bae HR: Time-dependent expression patterns of cardiac aquaporins following myocardial infarction. J Korean Med Sci 28: 402-408, 2013.
24. Wang Z, Wu G, Liu H, Xing N, Sun Y, Zhai Y, Yang B, Kong AT, Kuang $\mathrm{H}$ and Wang Q: Cardioprotective effect of the xanthones from Gentianella acuta against myocardial ischemia/reperfusion injury in isolated rat heart. Biomed Pharmacother 93: 626-635, 2017.

25. Kim JT, Chung HJ, Seo JY, Yang YI, Choi MY, Kim HI, Yang TH, Lee WJ, Youn YC, Kim HJ, et al: A fibrin-supported myocardial organ culture for isolation of cardiac stem cells via the recapitulation of cardiac homeostasis. Biomaterials 48: 66-83, 2015.

26. Bai M, Pan CL, Jiang GX, Zhang YM and Zhang Z: CircHIPK3 aggravates myocardial ischemia-reperfusion injury by binding to miRNA-124-3p. Eur Rev Med Pharmacol Sci 23: 10107-10114, 2019.

27. Wallert M, Ziegler M, Wang X, Maluenda A, Xu X, Yap ML, Witt R, Giles C, Kluge S, Hortmann M, et al: $\alpha$-Tocopherol preserves cardiac function by reducing oxidative stress and inflammation in ischemia/reperfusion injury. Redox Biol 26: 101292, 2019.

28. Wei B, Li WW, Ji J, Hu QH and Ji H: The cardioprotective effect of sodium tanshinone IIA sulfonate and the optimizing of therapeutic time window in myocardial ischemia/reperfusion injury in rats. Atherosclerosis 235: 318-327, 2014

29. Nakayoshi T, Sasaki K, Kajimoto H, Koiwaya H, Ohtsuka M, Ueno T, Chibana H, Itaya N, Sasaki M, Yokoyama S, et al: Correction: FOXO4-knockdown suppresses oxidative stress-induced apoptosis of early pro-angiogenic cells and augments their neovascularization capacities in ischemic limbs. PLoS One 10: e0127245, 2015

30. Nagy T, Kovács V, Hardi P, Veres TG, Takács I, Jancsó G, Sinay L, Fazekas G, Pintér Ö and Arató E: Inhibition of glutathione S-transferase by ethacrynic acid augments ischemia-reperfusion damage and apoptosis and attenuates the positive effect of ischemic postconditioning in a bilateral acute hindlimb ischemia rat model. J Vasc Res 52: 53-61, 2015.

31. Hassan M, Selimovic D, Hannig M, Haikel Y, Brodell RT and Megahed M: Endoplasmic reticulum stress-mediated pathways to both apoptosis and autophagy: Significance for melanoma treatment. World J Exp Med 5: 206-217, 2015

32. Lim YC, Budin SB, Othman F, Latip J and Zainalabidin S: Roselle polyphenols exert potent negative inotropic effects via modulation of intracellular calcium regulatory channels in isolated rat heart. Cardiovasc Toxicol 17: 251-259, 2017

33. Tao L, Huang K, Wang J, Xue Y, Zhou Y, He F, Shen Y, Wang J, $\mathrm{Gu} \mathrm{X}$, Ji K, et al: Retinol palmitate protects against myocardial ischemia/reperfusion injury via reducing oxidative stress and inhibiting apoptosis. Am J Transl Res 11: 1510-1520, 2019.

34. Pan C, Lou L, Huo Y, Singh G, Chen M, Zhang D, Wu A, Zhao M, Wang S and Li J: Salvianolic acid B and tanshinone IIA attenuate myocardial ischemia injury in mice by NO production through multiple pathways. Ther Adv Cardiovasc Dis 5: 99-111, 2011

35. Li S, Wu H, Han D, Zhang M, Li N, Yu W, Sun D, Sun Z, Ma S, Gao E, et al: ZP2495 protects against myocardial ischemia/reperfusion injury in diabetic mice through improvement of cardiac metabolism and mitochondrial function: The possible involvement of AMPK-FoxO3a signal pathway. Oxid Med Cell Longev 2018: 6451902, 2018.

36. Bhuiyan MS, Shibuya M, Shioda N, Moriguchi S, Kasahara J, Iwabuchi Y and Fukunaga K: Cytoprotective effect of bis(1-oxy-2-pyridinethiolato)oxovanadiun(IV) on myocardial ischemia/reperfusion injury elicits inhibition of Fas ligand and Bim expression and elevation of FLIP expression. Eur J Pharmacol 571: 180-188, 2007.

37. Shukla S, Sharma A, Pandey VK, Raisuddin S and Kakkar P. Concurrent acetylation of FoxO1/3a and p53 due to sirtuins inhibition elicit Bim/PUMA mediated mitochondrial dysfunction and apoptosis in berberine-treated HepG2 cells. Toxicol Appl Pharmacol 291: 70-83, 2016.

38. Babu PP, Suzuki G, Ono Y and Yoshida Y: Attenuation of ischemia and/or reperfusion injury during myocardial infarction using mild hypothermia in rats: An immunohistochemical study of Bcl-2, Bax, Bak and TUNEL. Pathol Int 54: 896-903, 2004.

39. Wang D, Hu X, Lee SH, Chen F, Jiang K, Tu Z, Liu Z, Du J, Wang L, Yin C, et al: Diabetes exacerbates myocardial ischemia/reperfusion injury by down-regulation of microRNA and up-regulation of O-GlcNAcylation. JACC Basic Transl Sci 3: 350-362, 2018

40. Yu LM, Dong X, Zhang J, Li Z, Xue XD, Wu HJ, Yang ZL, Yang Y and Wang HS: Naringenin attenuates myocardial ischemia-reperfusion injury via cGMP-PKGI $\alpha$ signaling and in vivo and in vitro studies. Oxid Med Cell Longev 2019: 7670854, 2019. 